\title{
Outcomes of Early versus Late Endotracheal Intubation in Patients with Initial Non-Shockable Rhythm Cardiopulmonary Arrest in the Emergency Department
}

\author{
Kiattichai Daorattanachai (D), Winchana Srivilaithon (D), Vitchapon Phakawan, \\ and Intanon Imsuwan $i D$ \\ Department of Emergency Medicine, Faculty of Medicine, Thammasat University, Pathum Thani 12120, Thailand \\ Correspondence should be addressed to Intanon Imsuwan; imsuwan2002@gmail.com
}

Received 20 June 2021; Revised 1 September 2021; Accepted 10 December 2021; Published 28 December 2021

Academic Editor: Kee Chong Ng

Copyright (c) 2021 Kiattichai Daorattanachai et al. This is an open access article distributed under the Creative Commons Attribution License, which permits unrestricted use, distribution, and reproduction in any medium, provided the original work is properly cited.

\begin{abstract}
Background. Sudden cardiac arrest is a critical condition in the emergency department (ED). Currently, there is no considerable evidence supporting the best time to complete advanced airway management (AAM) with endotracheal intubation in cardiac arrest patients presented with initial non-shockable cardiac rhythm. Objectives. To compare survival to hospital discharge and discharge with favorable neurological outcome between the ED cardiac arrest patients who have received AAM with endotracheal intubation within 2 minutes (early AAM group) and those over 2 minutes (late AAM group) after the start of chest compression in ED. Methods. We conducted a retrospective cohort study involving the ED cardiac arrest patients who presented with initial nonshockable rhythm in ED. Multivariable logistic regression analysis was used to evaluate the independent effect of early AAM on outcomes. The outcomes included the survival to hospital discharge and discharge with favorable neurological outcome. Results. There were 416 eligible participants: 209 in the early AAM group and 207 participants in the late AAM group. The early AAM group showed higher survival to hospital discharge compared with the late AAM group, but no statistically significant difference (adjusted odds ratio (aOR): 1.28, 95\% confidence interval $(\mathrm{CI}): 0.59-2.76, p=0.524)$. Discharge with favorable neurological outcome is also higher in the early AAM group (aOR: 1.68, 95\% CI, $0.52-5.45, p=0.387$ ). Conclusion. This study did not demonstrate a significant improvement of survival to hospital discharge and discharge with favorable neurological outcome in the ED cardiac arrest patients with initial non-shockable cardiac arrest who underwent early AAM within two minutes. More research is needed on the timing of AAM and on airway management strategies to improve survival.
\end{abstract}

\section{Introduction}

Cardiac arrest is a critical condition seen in emergency departments worldwide with incidence rates of 330,000 per year in the US and 275,000 in the European Union [1].

High-quality cardiopulmonary resuscitation (CPR), including effective chest compression, minimized interruption of compression, and appropriate assisted ventilation, is a crucial step in the survival of patients with cardiac arrest. Hypoxia is the most frequent cause of in-hospital cardiac arrest from a non-cardiac cause, approximately $20 \%$ of all causes $[2,3]$. Thus, ventilatory strategy is important during $\mathrm{CPR}$, especially in initial non-shockable are mostly caused by non-cardiac causes.

Bag-mask ventilation (BMV) with or without basic airway adjunct is commonly used as an initially assisted ventilation in cardiac arrest patients. Some patients need advanced airway management (AAM) with endotracheal intubation or insertion of a supraglottic airway device during CPR. The optimal method of ventilation during cardiac arrest remains controversial.According to the 2020 American Heart Association (AHA) guidelines for 
cardiopulmonary resuscitation and emergency cardiovascular care, BMV or an AAM may be considered during CPR in any setting [4].

Endotracheal intubation provides many advantages such as improved oxygenation, expel carbon dioxide, improved chest compression fraction, and measurement of end-tidal carbon dioxide for monitoring the quality of chest compression [5]. However, this procedure might increase the risk of interruption of chest compression, esophageal intubation, or undesirable hyperventilation [4].

The ED of Thammasat University Hospital uses advanced airway strategy with endotracheal intubation for resuscitation of cardiac arrests. Initial BMV in the first cycle of basic-life-support was followed by endotracheal intubation. Supraglottic airway devices, however, were only used in the failed intubation.

The previous study showed very high tracheal intubation success rates with $96.7 \%$ success within three attempts [6]. The present AHA guidelines recommend that, in the setting of high tracheal intubation success rates, advanced airway strategy can be used. However, no evidence currently exists demonstrating the optimal time to AAM. Wong et al. [7] revealed that AAM less than 5 minutes improves survival at discharge. Bobrow et al. showed the benefit of delayed AAM in the prehospital setting [8]. This study evaluated the effect of early AAM compared with late AAM in cardiac arrest patients presented with initial non-shockable cardiac rhythm.

\section{Methods}

This was a retrospective cohort study that was conducted in the emergency department of Thammasat University Hospital (TUH), Pathum Thani, Thailand, between January 2013 and October 2019. TUH is an 800-bed tertiary academic teaching hospital in the suburbs north of Bangkok, with approximately 1.1 million people living in the area with 60 , 000 patients visiting ED annually.

2.1. Participants. Eligible patients included all emergency department cardiac arrest (EDCA) patients over 18 years and presenting with initially non-shockable cardiac rhythm (asystole or pulseless electrical activity) who had an advanced airway placement during CPR. We excluded patients with do not attempt resuscitation (DNAR) orders, intubation prior to cardiac arrest, out-of-hospital intubation, and referral of the patient to another hospital after resuscitation.

2.2. Variables. We recorded data on a standard form, which included gender, age, initial location of cardiac arrest, etiology of cardiac arrest, initial cardiac rhythm, witnessed cardiac arrest, bystander CPR, collapsed time to first chest compression, time to first dose of epinephrine, time to AAM, defibrillation during resuscitation, and intravenous medications during resuscitation. AAM referred to endotracheal intubation, surgical cricothyroidotomy, and tracheostomy. Favorable neurological outcome was defined by cerebral performance category (CPC) 1 or 2. EDCA includes 3 groups of patients. (1) Out-of-hospital cardiac arrests who received prehospital CPR but without recovery and need to continue CPR when arrived at ED, (2) out-of-hospital cardiac arrests who did not receive prehospital CPR when arrived at ED, and (3) in-hospital cardiac arrests who received first chest compression in ED. Time to advanced airway management is the time from the first chest compression at ED to the successful placement of the advanced airway. Early AAM and late AAM were defined as the completion of advanced airway placement within two minutes and over two minutes, respectively.

2.3. Data Source/Measurement. Data were collected from Thammasat CPR Registry and the patient's medical charts. The outcomes were the survival to hospital discharge or within 30 days and the survival with favorable neurological outcome.

2.4. Study Size. From our pilot study in the emergency department, Thammasat University Hospital, survival to hospital discharge in early AAM and late AAM was $14 \%$ and $6 \%$, respectively. STATA software was used to calculate the sample size. To achieve $80 \%$ power to detect the difference, a sample size of 198 patients in each group was estimated for a 1 -sided test and an alpha level of $5 \%$.

2.5. Statistical Methods. Quantitative variables were presented as mean and standard variations. Categorial variables were expressed as percentages and compared using the chisquared test. Multivariable logistic regression analysis was used to evaluate the independent effect of early AAM on survival outcomes. Statistical significance was defined as a $p$ value $<0.05$. All analyses were performed using STATA software (version 14.0, StataCorp, College Station).

2.6. Ethics Approval. The study was approved by the Human Research Ethics Committee of Thammasat University, Faculty of Medicine (MTU-EC-EM-0-209/61).

\section{Results}

3.1. Participants. From January 2013 to October 2019, 805 patients with non-shockable cardiac arrest were identified. Four hundred and sixteen patients were eligible for the study, 209 patients $(50.24 \%)$ received AAM within two minutes, and 207 patients $(49.76 \%)$ received that over two minutes. 389 patients were excluded due to data missing (168), DNAR order (34), referral (27), and AAM before cardiac arrest or out-of-hospital advanced airway (160) (see Figure 1).

3.2. Descriptive Data. Clinical characteristics and demographic data are summarized in Table 1. The mean time of AAM in the early AAM group was 1.16 minutes (SD 0.83) compared with 5.87 minutes (SD 5.35) in the late AAM group.

The early AAM group received epinephrine earlier than the late AAM group (1.39 versus 2.28 minutes, $p<0.001$ ). There was no evidence of a statistical difference in gender, 


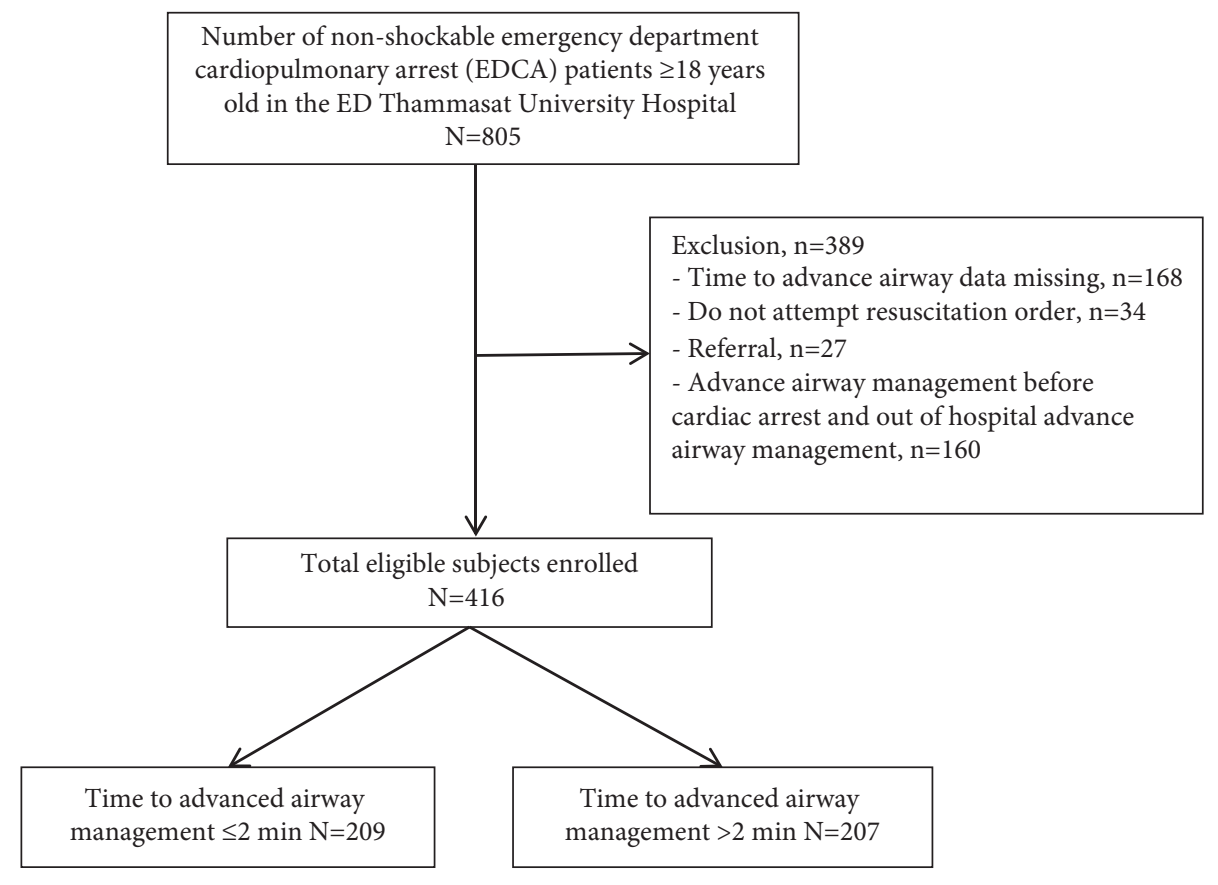

FIGURE 1: Flow diagram of the study population enrolled.

age, initial location of cardiac arrest, etiology of cardiac arrest, witnessed cardiac arrest, bystander CPR, collapsed time to first chest compression, initial rhythm of cardiac arrest, defibrillation during resuscitation, intravenous amiodarone used during resuscitation, and intravenous calcium administration.

3.3. Main Results. Return of spontaneous circulation (ROSC) occurred in 106 patients (50.72\%) in the early AAM group and 98 patients $(47.34 \%)$ in the late AAM group $(p=0.094)$. Survival to hospital discharge was 23 patients $(11 \%)$ in the early AAM group and 14 patients $(6.80 \%)$ in the late AAM group $(p=0.168)$. Discharge with favorable neurological outcome was 13 patients $(6.25 \%)$ and 6 patients (2.91\%) in the early AAM and the late AAM group, respectively $(p=0.157)$ (see Table 2$)$.

Multivariable logistic regression analysis was used to control the following variables: time to first dose epinephrine administration, sodium bicarbonate use, age, gender, etiology of cardiac arrest, and collapsed time to first chest compression. The early AAM group showed higher survival to hospital discharge compared with the late AAM group, but without statistically significant difference (adjusted odds ratio (aOR): 1.28, 95\% confidence interval (CI); 0.59-2.76, $p=0.524$ ) (see Table 3).

The early AAM group also had a higher favorable neurological outcome but without statistically significant difference (aOR: 1.68, 95\%CI; 0.52-5.45, $p=0.387$ ) (see Table 4).

\section{Discussion}

Early advanced airway management within two minutes after the first chest compression at ED in emergency department cardiac arrest patients presented with nonshockable cardiac rhythm showed higher survival to hospital discharge and survival with favorable neurological outcome compared with late over two minutes; however, there was no evidence of a significant difference between the study groups.

Although this study showed that a presumed cardiac etiology was the common cause of cardiac arrest (56\%), it was lower than the study from Izawa et al. (73.9\%) [9]. However, approximately $19 \%$ of all cardiac arrests were due to hypoxia, which is comparable with other studies $[2,3]$. The mean time of airway insertion was 1.6 minutes in the early AAM group while Wong et al. reported the mean time of 2.1 minutes in a group who were intubated before 5 minutes [7].

Even though the witnessed cardiac arrest was $71.11 \%$ (296/416), 10.6\% (44/416) of whom received bystander CPR. To date, the knowledge and skill in basic life support in the general population is generally low. The EMS system in Thailand is underdevelopment. The bystander CPR rate in our country is lower than the developed country. Therefore, time to first chest compression is longer than the developed country.

About half of our patients (49\%) had a return of spontaneous circulation, essentially the same as that reported by Wong et al. (50.3\%) [7]. Our overall survival to hospital discharge was $8 \%$ compared with $13.5 \%$ reported by Wang et al. [10].

Only $4.5 \%$ of the patients had discharge with favorable neurological outcome, whereas Wong et al. [7] had a better outcome at $11 \%$. We assume that the poorer outcome in our study could be explained by a non-cardiac cause of cardiac arrest (44\%) compared with another study (26.1\%) [9], which is usually associated with a poor prognosis.

The delayed administration of epinephrine is associated with a lower rate of survival in cardiac arrest with nonshockable rhythm [11]. The 2019 AHA Focused Update on 
TABLE 1: Study population characteristics.

\begin{tabular}{|c|c|c|c|c|c|}
\hline \multirow{3}{*}{ Patient characteristics } & \multicolumn{5}{|c|}{$\begin{array}{l}\text { All non-shockable rhythm cardiopulmonary arrest } \\
\text { patients }\end{array}$} \\
\hline & \multicolumn{2}{|c|}{$\begin{array}{l}\text { Time to advanced } \\
\text { airway } \\
\text { management } \\
\leq 2 \min (N=209)\end{array}$} & \multicolumn{2}{|c|}{$\begin{array}{c}\text { Time to advanced } \\
\text { airway } \\
\text { management } \\
>2 \text { min }(N=207)\end{array}$} & \multirow[t]{2}{*}{$p$ value } \\
\hline & $n$ & $\%$ & $\mathrm{n}$ & $\%$ & \\
\hline \multicolumn{6}{|l|}{ Gender } \\
\hline (i) Male & 125 & 49.60 & 127 & 50.40 & \multirow{2}{*}{0.764} \\
\hline (ii) Female & 84 & 51.22 & 80 & 48.78 & \\
\hline Age (years) mean (SD) & 62.32 & $(19.49)$ & 60.62 & $(17.99)$ & 0.358 \\
\hline \multicolumn{6}{|l|}{ Initial location of cardiac arrest } \\
\hline (i) Out of hospital & 166 & 50.46 & 163 & 49.54 & \multirow{2}{*}{0.904} \\
\hline (ii) In hospital & 43 & 49.43 & 44 & 50.57 & \\
\hline \multicolumn{6}{|l|}{ Etiology of cardiac arrest } \\
\hline (i) Presumed cardiac cause & 126 & 53.85 & 108 & 46.15 & \multirow{4}{*}{0.216} \\
\hline (ii) Trauma & 10 & 34.48 & 19 & 65.52 & \\
\hline (iii) Hypoxia & 39 & 49.58 & 40 & 50.63 & \\
\hline (iv) Other medical causes & 34 & 46.58 & 39 & 53.42 & \\
\hline Witnessed cardiac arrest & 151 & 51.01 & 145 & 48.99 & 0.666 \\
\hline Bystander CPR & 25 & 56.82 & 19 & 43.18 & 0.421 \\
\hline Collapsed time to first chest compression (minute) mean (SD) & 21.34 & $(24.46)$ & 25.97 & $(31.24)$ & 0.09 \\
\hline \multicolumn{6}{|l|}{ Initial rhythm of cardiac arrest } \\
\hline (i) Asystole & 135 & 48.56 & 143 & 51.44 & \multirow[t]{2}{*}{0.299} \\
\hline (ii) Pulseless electrical activity (PEA) & 74 & 54.01 & 63 & 45.99 & \\
\hline Time to first dose of epinephrine (minute) mean (SD) group (minute) & 1.39 & $(2.15)$ & 2.28 & $(2.69)$ & $<0.001$ \\
\hline (i) $0-2$ & 169 & 57.29 & 126 & 42.71 & $<0.001$ \\
\hline (ii) Over 2 & 38 & 32.20 & 80 & 67.80 & \\
\hline Time to advanced airway management mean (SD) & 1.16 & $(0.83)$ & 5.87 & $(5.35)$ & - \\
\hline Defibrillation during resuscitation & 53 & 48.62 & 56 & 51.38 & 0.738 \\
\hline Intravenous amiodarone used during resuscitation & 37 & 56.06 & 29 & 43.94 & 0.348 \\
\hline Intravenous calcium used during resuscitation & 56 & 46.67 & 64 & 53.33 & 0.387 \\
\hline Intravenous sodium bicarbonate used during resuscitation & 53 & 41.09 & 76 & 58.91 & 0.015 \\
\hline
\end{tabular}

TABLE 2: Outcome of resuscitation between time to advanced airway management $\leq 2$ minutes and $>2$ minutes.

\begin{tabular}{|c|c|c|c|c|c|}
\hline \multirow[t]{2}{*}{ Outcome } & \multicolumn{2}{|c|}{$\begin{array}{l}\text { Time to advanced } \\
\text { airway management } \\
\leq 2 \min (N=209)\end{array}$} & \multicolumn{2}{|c|}{$\begin{array}{c}\text { Time to advanced } \\
\text { airway } \\
\text { management } \\
>2 \min (N=207)\end{array}$} & \multirow[t]{2}{*}{$p$ value } \\
\hline & $\mathrm{n}$ & $\%$ & $\mathrm{n}$ & $\%$ & \\
\hline Sustained ROSC & $106 / 209$ & 50.72 & $98 / 207$ & 47.34 & 0.494 \\
\hline Survival to hospital discharge & $23 / 209$ & 11 & $14 / 207$ & 6.80 & 0.168 \\
\hline Survival to hospital discharge with favorable neurological outcome & $13 / 209$ & 6.25 & $6 / 207$ & 2.91 & 0.157 \\
\hline
\end{tabular}

TABLE 3: Odds ratio from multivariate logistic regression predicting survival to hospital discharge from time to advance airway management $\leq 2$ minutes VS $>2$ minutes.

\begin{tabular}{lcc}
\hline Associated factor & Odds ratio & 95\% confidence interval \\
\hline Time to advanced airway management $\leq 2 \mathrm{~min}^{*}$ & 1.28 & $0.59-2.76$ \\
Time to first dose of epinephrine within 2 min & 1.63 & $0.60-4.74$ \\
Intravenous sodium bicarbonate used during resuscitation & 0.21 & $0.06-0.74$ \\
Age & 0.99 & $0.98-1.02$ \\
Gender & 1.40 & $0.65-3.01$ \\
Etiology of cardiac arrest & 0.93 & $0.68-1.28$ \\
Collapsed time to first chest compression & 0.95 & $0.93-0.98$ \\
\hline
\end{tabular}

${ }^{*}$ Reference with time to advanced airway management $>2$ minutes. 
TABLE 4: Odds ratio from multivariable logistic regression predicting survival to hospital discharge with favorable neurological outcome from time to advance airway management $\leq 2$ minutes and $>2$ minutes.

\begin{tabular}{|c|c|c|c|}
\hline Associated factor & Odds ratio & $95 \%$ confidence interval & $p$ value \\
\hline Time to advanced airway management $\leq 2$ min* $^{*}$ & 1.68 & $0.52-5.45$ & 0.387 \\
\hline Time to first dose of epinephrine within 2 minutes & 0.92 & $0.23-3.73$ & 0.904 \\
\hline Intravenous sodium bicarbonate used during resuscitation & 0.14 & $0.02-1.11$ & 0.063 \\
\hline Age & 0.99 & $0.96-1.02$ & 0.536 \\
\hline Gender & 1.74 & $0.57-5.28$ & 0.328 \\
\hline Etiology of cardiac arrest & 0.61 & $0.36-1.04$ & 0.068 \\
\hline Collapsed time to first chest compression & 0.31 & $0.86-0.97$ & 0.003 \\
\hline
\end{tabular}

${ }^{*}$ Reference with time to advanced airway management $>2 \mathrm{~min}$.

Advanced Cardiovascular Life Support [12] emphasized the early epinephrine administration in non-shockable cardiac arrest. From our study, early epinephrine administration was associated with early AAM. Izawa et al. [9] demonstrated that in their early AAM group, their patients also received early epinephrine administration. In contrast, Lupton et al. [13] did not find an association between AAM and time to initial epinephrine administration in out-ofhospital cardiac arrest. This finding might be explained by the effect of high performance of the CPR team; with more effective resuscitation, epinephrine can be administered immediately, and the advanced airway can be also placed early.

In our study, we demonstrated a higher odds ratio of survival to hospital discharge, ROSC, and neurological outcome in the early AAM group, but none were statistically significant. During the circulatory phase of CPR, early AAM might be beneficial for a favorable outcome [14] but controversy remains regarding the potentially beneficial effects of advanced airway management on neurological outcome.

Wong et al. [7] demonstrated that AAM before 5 minutes was associated with a better survival rate at hospital discharge (16.16 versus 11\%). Better neurological outcomes were reported by Izawa et al. [9] (early AAM 2.2\% versus late AAM 1.4\%, aOR: 1.58 (95\% CI; 1.24-2.02)) and Kajino et al. [15] with early prehospital AAM performed by EMS personnel (aOR for one minute delay, $0.91,95 \% \mathrm{CI} ; 0.88$ to 0.95).

A poor neurological outcome was reported by Wang et al. [10] in delayed AAM in hospitalized patients with a non-shockable rhythm (OR: 0.86, 95\% CI; 0.80-0.93, $p<0.001)$. On the contrary, Hasegawa et al. [16] showed that a poor neurological outcome was more likely with AAM compared to conventional BMV (1.1\% versus $2.9 \%$, unadjusted OR: $0.38,95 \%$ CI; 0.36 to 0.39 ). Andersen et al. [17] revealed that tracheal intubation during the first 15 minutes had lower rate of hospital discharge. According to the 2020 AHA guidelines for cardiopulmonary resuscitation and emergency cardiovascular care [4], there is no evidence that AAM is better than BMV.

Early AAM allows for high-quality CPR monitoring such as end-tidal carbon dioxide monitoring and asynchronous chest compression [18] and is associated with less gastric insufflation and aspiration. Moreover, it contributes oxygenation and ventilation control which may improve the outcome $[5,19]$. In contrast to AAM, endotracheal tube intubation may interrupt chest compression, be poorly placed or inserted into the esophagus, and increase the risk of hyperventilation [20]. However, these adverse events would be diminished by an experienced physician and proper monitoring. Visualizing chest movements and chest auscultation, listening over epigastrium, and $\mathrm{EtCO}_{2}$ monitoring should be done immediately after intubation during CPR to ensure optimal CPR quality [12]. Training should be held frequently for physicians who perform intubation to improve tracheal intubation success rate, decrease interruption of chest compression, and reduce complications [4].

We emphasized the crucial role of high-quality CPR over advanced airway management. Interruption of chest compression should be minimized during resuscitation. Initiation of intubation should follow high-quality CPR.

4.1. Limitation. Our study had several limitations. First, the sample size was relatively small that reduced statistical power to detect small differences. We had to exclude a quarter of patients because of missing important data, which is the disadvantage of a retrospective study. Second, the rate and depth of chest compression were not recorded in the hospital notes nor the difficulty and success rate in achieving AAM. Finally, a supraglottic airway device was not used in any of our patients.

\section{Conclusions}

Our retrospective study did not demonstrate a significant improvement of survival to hospital discharge and survival with favorable neurological outcome in the patients with initial non-shockable cardiac arrest who underwent early AAM within two minutes. More research is needed on the timing of AAM and on airway management strategies to improve survival.

\section{Data Availability}

The data used to support the findings of this study are available from the corresponding author upon request.

\section{Disclosure}

This study is part of the project from the Research Group in Emergency Medicine and Emergency Critical Care of the Faculty of Medicine of Thammasat University. 


\section{Conflicts of Interest}

The authors declare that they have no conflicts of interest.

\section{Authors' Contributions}

$\mathrm{KD}$, WS, and II were responsible for conceptualization and methodology. KD and VP were responsible for data curation and investigation. KD and WS were responsible for formal analysis. KD and II were responsible for supervision, validation, and review and editing. KD, VP, and II were responsible for original draft preparation. All authors have read and agreed to the published version of the manuscript.

\section{References}

[1] J. Berdowski, R. A. Berg, J. G. P. Tijssen, and R. W. Koster, "Global incidences of out-of-hospital cardiac arrest and survival rates: systematic review of 67 prospective studies," Resuscitation, vol. 81, no. 11, pp. 1479-1487, 2010.

[2] D. Bergum, T. Nordseth, O. C. Mjølstad, E. Skogvoll, and B. O. Haugen, "Causes of in-hospital cardiac arrest-incidences and rate of recognition," Resuscitation, vol. 87, pp. 63-68, 2015.

[3] W. Srivilaithon, K. Amnuaypattanapon, C. Limjindaporn et al., "Predictors of in-hospital cardiac arrest within $24 \mathrm{~h}$ after emergency department triage: a case-control study in urban Thailand," Emergency Medicine Australasia, vol. 31, no. 5, pp. 843-850, 2019.

[4] A. R. Panchal, J. A. Bartos, J. G. Cabañas et al., "Part 3: adult basic and advanced life support: 2020 American heart association guidelines for cardiopulmonary resuscitation and emergency cardiovascular care," Circulation, vol. 142, no. 16, pp. S366-S468, 2020.

[5] J. L. Benoit, D. K. Prince, and H. E. Wang, "Mechanisms linking advanced airway management and cardiac arrest outcomes," Resuscitation, vol. 93, pp. 124-127, 2015.

[6] W. Srivilaithon, "Prospective observational study of emergency airway management in emergency department," Medical Journal of the Medical Association of Thailand, vol. 99, no. 4, pp. S131-S137, 2016.

[7] M. L. Wong, S. Carey, T. J. Mader, and H. E. Wang, "Time to invasive airway placement and resuscitation outcomes after inhospital cardiopulmonary arrest," Resuscitation, vol. 81, no. 2, pp. 182-186, 2010.

[8] B. J. Bobrow, L. L. Clark, G. A. Ewy et al., "Minimally interrupted cardiac resuscitation by emergency medical services for out-of-hospital cardiac arrest," JAMA, vol. 299, no. 10, pp. 1158-1165, 2008.

[9] J. Izawa, T. Iwami, K. Gibo et al., “Timing of advanced airway management by emergency medical services personnel following out-of-hospital cardiac arrest: a population-based cohort study," Resuscitation, vol. 128, pp. 16-23, 2018.

[10] C.-H. Wang, W.-J. Chen, W.-T. Chang et al., "The association between timing of tracheal intubation and outcomes of adult in-hospital cardiac arrest: a retrospective cohort study," Resuscitation, vol. 105, pp. 59-65, 2016.

[11] R. Khera, P. S. Chan, M. Donnino, and S. Girotra, "Hospital variation in time to epinephrine for nonshockable in-hospital cardiac arrest," Circulation, vol. 134, no. 25, pp. 2105-2114, 2016.

[12] A. R. Panchal, K. M. Berg, K. G. Hirsch et al., “American heart association focused update on advanced cardiovascular life support: use of advanced airways, vasopressors, and extracorporeal cardiopulmonary resuscitation during cardiac arrest: an update to the American heart association guidelines for cardiopulmonary resuscitation and emergency cardiovascular care," Circulation, vol. 140, no. 24, pp. e881-e94, 2019.

[13] J. R. Lupton, R. Schmicker, M. R. Daya et al., "Effect of initial airway strategy on time to epinephrine administration in patients with out-of-hospital cardiac arrest," Resuscitation, vol. 139, pp. 314-320, 2019.

[14] M. L. Weisfeldt and L. B. Becker, "Resuscitation after cardiac arrest," JAMA, vol. 288, no. 23, pp. 3035-3038, 2002.

[15] K. Kajino, T. Iwami, T. Kitamura et al., "Comparison of supraglottic airway versus endotracheal intubation for the pre-hospital treatment of out-of-hospital cardiac arrest," Critical Care (London, England), vol. 15, no. 5, Article ID R236, 2011.

[16] K. Hasegawa, A. Hiraide, Y. Chang, and D. F. M. Brown, "Association of prehospital advanced airway management with neurologic outcome and survival in patients with out-ofhospital cardiac arrest," JAMA, vol. 309, no. 3, pp. 257-266, 2013.

[17] L. W. Andersen, A. Granfeldt, C. W. Callaway et al., "Association between tracheal intubation during adult in-hospital cardiac arrest and survival," JAMA, vol. 317, no. 5, pp. 494-506, 2017.

[18] J. Yeung, M. Chilwan, R. Field, R. Davies, F. Gao, and G. D. Perkins, "The impact of airway management on quality of cardiopulmonary resuscitation: an observational study in patients during cardiac arrest," Resuscitation, vol. 85, no. 7, pp. 898-904, 2014.

[19] W. Spindelboeck, O. Schindler, A. Moser et al., "Increasing arterial oxygen partial pressure during cardiopulmonary resuscitation is associated with improved rates of hospital admission," Resuscitation, vol. 84, no. 6, pp. 770-775, 2013.

[20] T. P. Aufderheide, G. Sigurdsson, R. G. Pirrallo et al., "Hyperventilation-induced hypotension during cardiopulmonary resuscitation," Circulation, vol. 109, no. 16, pp. 1960-1965, 2004. 